\title{
EVALUACIÓN DE LA GESTIÓN EN EL PARQUE REGIONAL DE LAS SALINAS Y ARENALES DE SAN PEDRO DEL PINATAR (MURCIA)
}

\author{
Gustavo A. Ballesteros Pelegrín. \\ Dpto. de Geografía \\ Universidad de Murcia
}

\section{RESUMEN}

El Parque Regional de las Salinas y Arenales de San Pedro del Pinatar es un territorio multifuncional desde el punto de vista social, económico y ambiental, donde es imposible separar y, por tanto gestionar de forma independiente, las tramas paisajísticas y ecológicas de las tramas socioeconómicas. Para su gestión, la Administración se apoya en el Plan de Ordenación de los Recursos Naturales aprobado en 1995, que sin embargo ha sido ejecutado en el 50 \% de su articulado. Se desarrollan diversas iniciativas de conservación de fauna y flora silvestre, recogida de residuos sólidos, una adecuada información al visitante y mejora del conocimiento de los diferentes elementos de los espacios protegido. Sin embargo la participación social es escasa, la Junta Rectora se ha reunido en solo cuatro ocasiones, la última en 2001, se han producido desarrollos urbanísticos en la periferia del Parque que producen deterioro paisajístico y se han desarrollado una serie de infraestructuras que han reducido en unas 7 hectáreas la superficie protegida.

Palabras clave: Gestión, planificación, conservación, información, investigación, deterioro, paisaje.

\section{ABSTRACT}

\section{Evaluation of Management Plan on San Pedro del Pinatar saltworks and sandy areas Regional Park (Murcia, Spain)}

The Regional Park of Salinas y Arenales de San Pedro del Pinatar is a multifunctional territory from the standpoint of social, economic and environmental, where it is impossible to separate and therefore independently manage, ecological landscape and socioeconomic frames . For its management, Regional Public Administration supports the Natural Resources Management Plan approved in 1995, which has been implemented in 50\% only. There are several development conservation wildlife initiatives on going related to solid waste collection, adequate information to visitors and better knowledge of protected areas essentials. However, public participation is low, the Governing Board has met only four times. The last time was far in 2001. Urban sprawl had occurred in park surroundings with landscape deterioration effects and had reduced 7 hectares of initially declared protected area.

Key words: Management, Planning, conservation, information, research, habitat and landscape loss.

\section{LA EVALUACIÓN DE LA PLANIFICACIÓN Y GESTIÓN EN ESPACIOS PROTEGIDOS}

La evaluación de la gestión se ha convertido en uno de los grandes retos a los que se enfrentan los espacios naturales protegidos. La gran importancia que estos lugares han adquirido como herramientas de conservación de la naturaleza en todo el mundo (UNEP-WCMC, 2008), han traído un creciente interés por conocer y comunicar cuáles son los resultados obtenidos y en qué medida las áreas protegidas sirven para alcanzar los objetivos para los que se concibieron (De Lucio et al., 2010).

Aunque hay pocas dudas de que globalmente las áreas protegidas acogen una parte importante de la biodiversidad y amortiguan los procesos que las amenazan, planteando modelos de desarrollo sostenible con las actividades económicas y sociales que en ellos se desarrollan, el conocimiento científico sobre la eficacia del mantenimiento de la biodiversidad con la necesaria compatibilidad con las actividades

Contacto: gabpl@um.es 
humanas en muchos casos es insuficiente (Gaston et al., 2008 y De Lucio et al., 2010), y la gestión se basa en ocasiones más en criterios empíricos que en la evidencia científica (Pullin, 2004).

La evaluación de la gestión es definida como el "juicio del grado en que se han alcanzado determinados objetivos establecidos de antemano, entre los que pueden considerarse los objetivos para los que se ha creado un espacio protegido". Esta definición de evaluación supone un marco de referencia muy amplio, ya que los objetivos por los que se designan áreas protegidas pueden ser muy variados, y a menudo las áreas protegidas se caracterizan por la producción de bienes y servicios de difícil valoración.

Dentro de la evaluación de la gestión cabe matizar además si se evalúa la "eficacia", es decir, si la gestión ha alcanzado determinados objetivos, o bien la "eficiencia" cuando además se hace referencia al coste asumido para conseguir dichos objetivos; la eficiencia implica eficacia con economía de medios. Algunos autores proponen además la "efectividad" como término que englobaría la eficacia y eficiencia. (Mallarach et al., 2008).

La evaluación de la gestión de las áreas protegidas comprende entonces aspectos tan diversos como su designación, la adecuación y suficiencia de los medios dedicados a la gestión, la valoración de los propios procesos de gestión, hasta el alcance de los objetivos de los instrumentos de planificación y gestión, incluyendo la conservación de los valores por los que se designaron las áreas protegidas (De Lucio et al., 2010).

La planificación y gestión de áreas protegidas se pueden plantear de múltiples formas, pero se pueden resumir en dos categorías genéricas con implicaciones diferentes: aquellas en las que el énfasis se pone en la protección del mundo natural y aquellas otras en las que está en el mantenimiento de una relación entre la naturaleza y las actividades económicas de la gente que dependen de éstas. Es esta segunda categoría la que nos interesa especialmente por el alcance y por su relevancia en este Parque Regional, que el Consejo de Europa (2000) como "área, cuyo carácter es el resultado de la acción e interacción de los factores humanos y naturales".

Los parques naturales -o parques regionales- surgen como consecuencia de la interacción del hombre con el medio ambiente que le rodea. Es evidente que no es el "paisaje", o determinados aspectos de la "biodiversidad" los únicos objetos de protección, hay muchos elementos de carácter económico y social que se encuentran encerrados en estos espacios y que contribuyen a su mantenimiento y diversidad en el sentido más amplio de la palabra. Por tanto, en un parque natural -o regional- están representadas: la naturaleza, las personas, la historia y los atributos físicos, así como los valores sociales y culturales.

Respecto a las políticas relativas al rol de los actores locales, hacen referencia a aquellos que su medio de vida está íntimamente conectado con el parque y/o con los valores naturales y culturales que se pretenden conservar. Situarlos en primer lugar no significa que el medio ambiente y otros valores deban estar subordinados a los factores económicos y sociales, sino reconocer que la supervivencia de estos valores depende del apoyo de los habitantes, y que la gestión debe incorporar, en la medida de lo posible, las necesidades e inquietudes de los habitantes del parque regional.

Junto a las cuestiones relativas a las especies, ecosistemas y lugares que requieren protección, en equilibrio con las actividades económicas, sociales y culturales que en estos espacios se desarrollan, comienzan a surgir preguntas sobre el funcionamiento de las áreas protegidas: ¿Cuáles son los objetivos de conservación y promoción del desarrollo sostenible? ¿Cómo medir el progreso en el alcance de éstos? ¿Es eficaz el manejo de áreas protegidas? y en definitiva ¿Qué podemos entender por eficacia en términos de conservación?.

Según De Lucio et al., (2010), en España existen pocas iniciativas de evaluación de la gestión de áreas protegidas. Generalmente se trata de evaluaciones contables y de actividades, recogidas en memorias anuales de gestión (Mallarach, 2008).

Se han realizado algunas evaluaciones de sistemas de áreas protegidas en distintas regiones, promovidas por la propia administración, como por ejemplo la Junta de Andalucía (1999), el Gobierno de Aragón (DGA, 2004) o la Junta de Castilla y León (Consejo Regional de Espacios Naturales Protegidos de Castilla y León, 2007). Entre las escasas evaluaciones objetivas y comprensivas de los sistema de espacios protegidos realizada por la entidad independiente de evaluación del Plan de Espacios de Interés Natural de Cataluña, destaca la realizada por la Sociedad Catalana de Historia Natural (Mallarach et al., 2008).

Pueden citarse además algunas iniciativas de evaluación de áreas protegidas basadas en indicadores cuantitativos recopilados de forma sistemática y continuada. Es el caso del sistema de indicadores para la evaluación de la Reserva de la Biosfera de Menorca, a cargo del Observatorio Socioambiental de Menorca o el sistema de indicadores de seguimiento y evaluación para los espacios naturales protegidos de la Cordillera Cantábrica, desarrollado por la administración asturiana (García y Huergo, 2007). 
Si descontamos estas iniciativas más o menos aisladas, en general los avances en los últimos años han sido escasos (De Lucio et al., 2010), aunque diversos compromisos nacionales e internacionales establecen la necesidad de evaluar las áreas protegidas, tal es el caso de la Red Natural 2000, que implica la exigencia a los Estados miembros de la Unión Europea de la necesidad de establecer sistemas de seguimiento, y de evaluar e informar de forma periódica sobre el estado de conservación de las especies y hábitats de interés (Artículos 11 y 17 de la Directiva Hábitats).

\section{EVALUACIÓN DE LA PLANIFICACIÓN Y GESTIÓN EN EL PARQUE REGIONAL DE LAS SALINAS Y ARENALES DE SAN PEDRO DEL PINATAR}

\subsection{Aspectos generales}

El Parque Regional se localizan en la porción litoral más septentrional de la Región de Murcia, en los términos municipales de San Pedro del Pinatar y San Javier. Tiene una superficie de 496 hectáreas, limita al norte con la urbanización del Mojón; al sur con la Manga del Mar Menor; al este con el Mar Mediterráneo y al oeste con el Mar Menor y la urbanización de Lo Pagán (figura 1).

Figura 1. Localización del Parque Regional de las Salinas y Arenales de San Pedro del Pinatar.

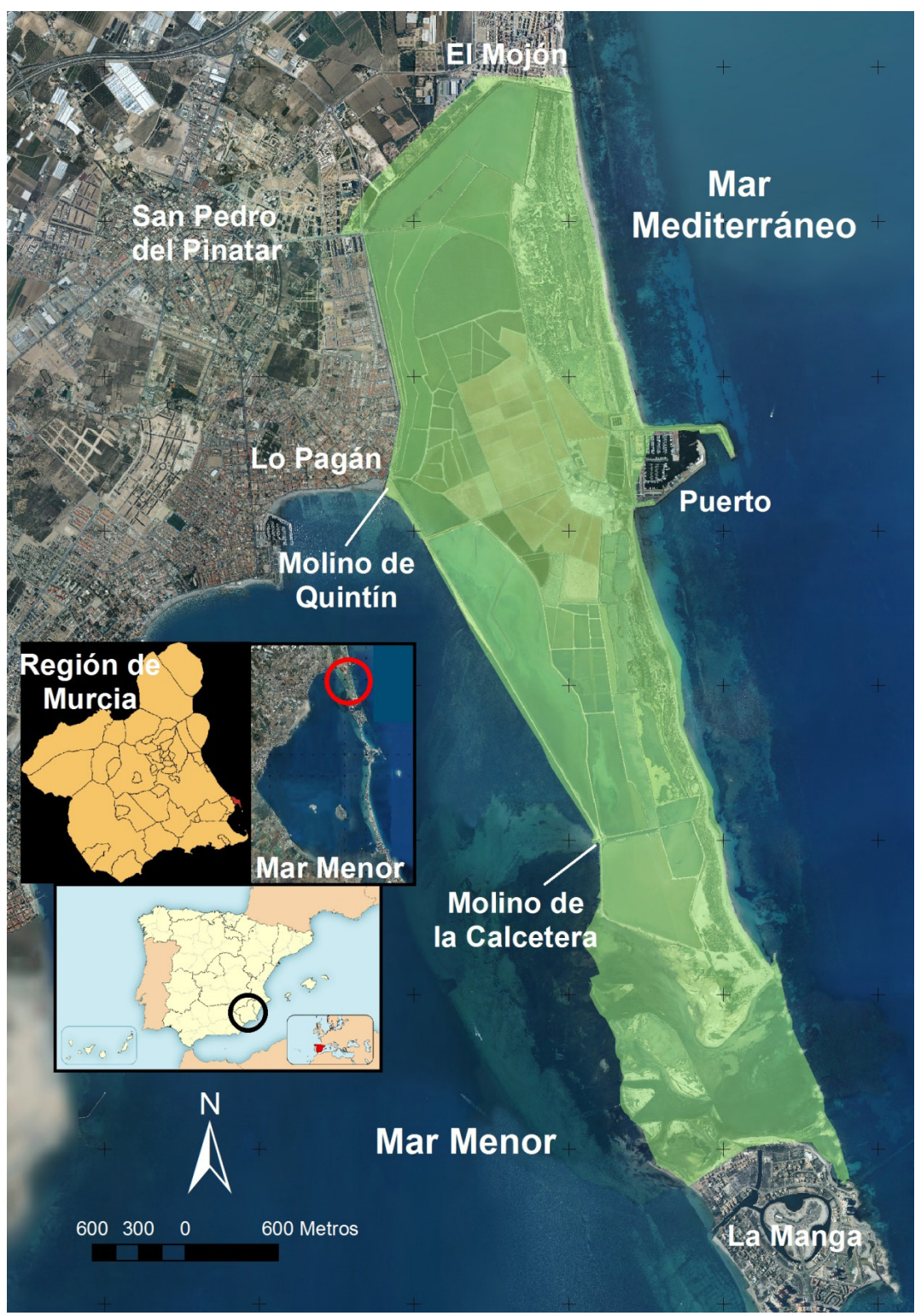

Elaboración propia. 
Constituye el complejo de humedales más importante del Mar Menor, formado principalmente por extensas superficies destinadas a la explotación salinera y sistemas ecológicos adyacentes de gran interés, típicos de zonas palustres y de otros ecosistemas sedimentarios: saladares, arenales y playas, que junto con las encañizadas albergan las mayores concentraciones regionales de aves acuáticas durante todo el año.

En términos generales se trata de un espacio con vocación de conservación de la avifauna, mantenida en buena parte por la actividad salinera, que ha utilizado técnicas tradicionales de explotación permitiendo la conservación de sus valores naturales. Presenta además un carácter de espacio periurbano manifiesto, dada su situación geográfica y el enorme desarrollo de las iniciativas urbano-turísticas en su entorno.

Ha sido incluido en dos Convenios Internacionales: la Lista de Humedales de Importancia Internacional del Convenio RAMSAR, junto a la laguna del Mar Menor y en aplicación del protocolo sobre Zonas Especialmente Protegidas de Importancia para el Mediterráneo (Convenio de Barcelona), se declara en 2001 la ZEPIM, Área del Mar Menor y Zona Oriental Mediterránea de la Costa de la Región de Murcia. En aplicación de la normativa de la Unión Europea, fue declarado como Zona de Especial Protección para las Aves (ZEPA) y Lugar de Importancia Comunitaria (LIC), por lo que forma parte de la Red Natura 2000 y en base a la legislación de la Región de Murcia fue declarado como Parque Regional y dispone de un Plan de Ordenación de los Recursos Naturales (PORN) aprobado mediante Decreto en 1995.

Dentro del Parque acceden a diario más de 300 trabajadores (tabla 1) para desarrollar su actividad profesional en empresas privadas, algunas inciden directamente en su conservación como la explotación salinera y la explotación pesquera de las encañizadas y otras se han desarrollado en torno al puerto de San Pedro del Pinatar, como es la flota pesquera, el cultivo de granjas de diferentes especies de peces marinos que se desarrollan a una milla frente a dicho Puerto, puertos deportivos y el desarrollo del turismo. A lo que hay que sumar las actividades que desarrollan las diferentes administraciones de investigación acuícola, información y conservación del Parque, de protección civil y cruz roja.

Tabla 1. Trabajadores que desarrollan su actividad dentro del Parque Regional de Las Salinas y Arenales de San Pedro del Pinatar

\begin{tabular}{|l|c|c|c|c|c|c|}
\hline \multirow{2}{*}{ EMPLEO EN EL PARQUE REGIONAL } & \multicolumn{2}{|c|}{1996} & \multicolumn{2}{|c|}{2003} & \multicolumn{2}{c|}{2010} \\
\cline { 2 - 7 } & Verano & $\begin{array}{c}\text { Resto } \\
\text { del año }\end{array}$ & Verano & $\begin{array}{c}\text { Resto } \\
\text { del año }\end{array}$ & $\begin{array}{c}\text { Verano } \\
\text { del año }\end{array}$ \\
\hline Empresas privadas & +293 & +289 & 364 & 349 & 324 & 312 \\
\hline Administraciones públicas & 44 & 19 & 55 & 26 & 39 & 16 \\
\hline Total empleo dentro del Parque Regional & +337 & +308 & 419 & 375 & 363 & 328 \\
\hline
\end{tabular}

Fuente: Dirección General del Medio Natural (1997 y 2003); López et al., (2010) y datos suministrados por Salinera Española y otros. Elaboración propia.

\subsection{Recursos humanos y materiales para la gestión}

\subsubsection{Recursos humanos}

El Parque Regional cuenta con un equipo de unos 8 profesionales en 2010 que trabajan para su gestión, mantenimiento y conservación (Biocyma, 2010).

\subsubsection{Recursos materiales}

En la tabla 2 se señalan las principales instalaciones de gestión de uso público.

Tabla 2. Instalaciones de gestión y uso público en el Parque Regional de Las Salinas y Arenales de San Pedro del Pinatar (2010)

\begin{tabular}{|l|l|}
\hline \multicolumn{1}{|c|}{ TIPO DE INSTALACIÓN } & \multicolumn{1}{c|}{ EQUIPAMIENTO } \\
\hline $\begin{array}{l}\text { Información, atención al visitante y } \\
\text { comunicación social }\end{array}$ & $\begin{array}{l}\text { Centro de Visitantes "Las Salinas". } \\
\text { Punto de Información "Charca de El Coterillo". }\end{array}$ \\
\hline Áreas Recreativas & Centro de Visitantes "Las Salinas" y Charca de "El Coterillo" \\
\hline Observatorios & $\begin{array}{l}\text { Torre pinar de "El Coterillo", Centro de Visitantes "Las Salinas", charca de "El Coterillo" (2) } \\
\text { y charca del Centro de Visitantes (2). }\end{array}$ \\
\hline Itinerarios auto guiados & Senda de la Encañizada y Senda de El Coterillo. \\
\hline Almacén & Edificación tipo cochera 50m² junto al Centro de Visitantes \\
\hline Aseos & Aseos portátiles en la Mota de los Molinos. \\
\hline Aparcamiento & $\begin{array}{l}\text { Centro de Visitantes "Las Salinas" y charca de "El Coterillo", Curva de la Culebra, frente al } \\
\text { puerto y playa de la Llana. }\end{array}$ \\
\hline
\end{tabular}

Fuente: Biocyma (2010). 


\subsection{Clasificación de las acciones de gestión}

\subsubsection{Tipos de acciones}

La clasificación de las acciones de gestión es una tarea compleja. Según De Lucio et al., (2010), a menudo es difícil decidir a qué categoría corresponde una acción determinada que puede satisfacer varios objetivos complementarios.

Tomando como base las discusiones del Grupo de Conservación de Europarc-España y el análisis de las categorías utilizadas de gestión de diferentes administraciones, De Lucio et al., (2010), propone una clasificación que ha sido adaptada a la realidad de éste Parque:

1. Funcionamiento básico del Parque: Desarrollo de los instrumentos de ordenación y planificación (PORN y PRUG), mantenimiento de las instalaciones, limpieza y recogida de residuos, vigilancia y gestión preventiva y expedientes sancionadores.

2. Gestión "proactiva": Conservación de especies amenazadas y acciones de conservación Ex-situ, mediante la recuperación de fauna herida o enferma en el Centro de Recuperación de Fauna Silvestre. Conservación del paisaje y del hábitat mediante la restauración de procesos para recuperar ecosistemas y la eliminación y control de especies de flora invasora.

3. Gestión del uso público y educación ambiental: mejora de infraestructuras de atención al visitante, evolución del número de visitantes atendidos e itinerarios guiados, aula de naturaleza, encuestas a los visitantes sobre sus intereses, necesidades y satisfacción, certificación de la Q de Calidad Turística, quejas, sugerencias y felicitaciones.

4. Mejora del conocimiento: Información básica estudios promovidos desde la Administración y colaboración en otros proyectos, seguimiento de especies y hábitats.

5. Participación social: Junta Rectora.

6. Dinamización social: Difusión de actividades participando en medios de comunicación locales y regionales, colaboración con otras administraciones empresas y organizaciones. Formación del capital humano del Parque mediante el desarrollo de cursos de formación.

\subsubsection{Funcionamiento básico}

El funcionamiento básico está directamente relacionado con el trabajo diario de aplicación y desarrollo de los instrumentos de ordenación y planificación, así como de administración y mantenimiento y vigilancia.

\subsubsection{Desarrollo de los instrumentos de ordenación y planificación}

Plan de Ordenación de los Recursos Naturales (PORN)

El PORN de las Salinas y Arenales de San Pedro del Pinatar, fue aprobado por el R.D. no 44/1995, de 26.05.1995 y publicado en el BORM n ${ }^{\circ}$ 151, de 01.07.1995. En los 108 artículos que lo componen, se contabiliza un total de 48 compromisos para su desarrollo, que han tenido una desigual ejecución y cumplimiento. El articulado del PORN ha sido desarrollado desde su aprobación en 1995 y hasta diciembre de 2010 en un $48 \%$, un $2 \%$ dispone de un desarrollo parcial y el $50 \%$ restante está pendiente de ser aplicado

Entre los artículos que están siendo ejecutados y que han tenido una especial relevancia en el desarrollo socioeconómico y/o en la conservación del Parque Regional, se encuentra el artículo 22.3., donde los sucesivos y continuados planes de control de expansión de especies exóticas puestos en marcha desde inicios de los años 90, ha conseguido reducir de forma significativa el área de distribución de la pitera (Agave americana) y el carpobrotus (Carpobrotus edulis), mejorando la calidad paisajística y el hábitat del ecosistema dunar. Asimismo, el artículo 23.3, referente a la puesta en marcha de una red de itinerarios y recorridos que abarca todo el Parque, ha permitido que un número cada vez mayor de visitantes puedan conocerlo. La aplicación del artículo 103.1 a), permite disponer de un Servicio de Información al Visitante con profesionales muy cualificados, y de un Centro de Interpretación bien dotado que satisface las necesidades de los visitantes. Con el desarrollo del artículo 105.5, se ha priorizado que el equipo de información y otros trabajadores del Parque procedan de las poblaciones de entorno del mismo Parque (San Pedro del Pinatar y San Javier), y con el apartado 107.1. del PORN referente a la "recuperación 
de la Actividad Pesquera de las Encañizadas", se ha logrado compatibilizar una actividad económica tradicional con la conservación y mejora de éste sector del Parque.

Por otro lado, entre los artículos que no se han puesto en marcha que podrían ser relevantes en el desarrollo socioeconómico y/o en la conservación del Parque Regional, destaca la redacción y aplicación de los planes de recuperación y conservación de la fauna y flora (artículos 12.2 y 13.2). La recuperación y conservación de elementos de Interés Cultural, como son los molinos de Quintín y de la Calcetera, mediante el acuerdo con organismos públicos/privados (artículo 16.3), así como solicitar a la Consejería de Cultura incoar determinados expedientes de declaración de BIC para otros edificios con valores patrimoniales relevantes (artículo 16.5.). La aplicación del artículo 83.1, en el sentido de limitar la velocidad a $40 \mathrm{Km} / \mathrm{h}$. reduciría la elevada mortandad de vertebrados en la carretera de acceso a Puerto. También se debería asignar un presupuesto anual para el desarrollo del PRUG (artículo 90), una vez que sea aprobado (artículo 93.1). Fomentar la participación pública mediante reuniones anuales de la Junta Rectora (artículo 92). Así mismo, disponer de un Convenio con la Compañía Salinera (artículo 106), es una de las principales tareas que de forma inexplicable aún pendiente de acometer, dado que unas 2/3 partes del Parque forman parte de la explotación industrial, su relevancia económica y social, se convierte en el actor y eje principal que incide en la conservación de este espacio protegido. Finalmente, evaluar la posibilidad de exención de tasas y bonificación de impuestos a propietarios y actividades siempre y cuando estas actividades incidan de forma positiva en su conservación (artículo 108), tal y como es la explotación industrial salinera y la explotación pesquera tradicional de las Encañizadas.

Plan Rector de Uso y Gestión (PRUG)

El PRUG es el instrumento donde se fijan las normas generales de uso y gestión del Parque, es el principal instrumento de desarrollo del PORN, regulando los usos y actividades, estableciendo las normas y procedimientos de gestión y conservación, investigación, uso público, etc.

El artículo 89 del PORN, incluido en la Sección I: Directrices generales para la planificación y gestión, señala entre otros aspectos, que "en el plazo de un año a partir de la aprobación definitiva del PORN, se elaborará el PRUG".

Los artículos 93 y 94, incluidos en la Sección II: Directrices para la elaboración del Plan Rector de Uso y Gestión, señalan que el PRUG puede redefinir en detalle la zonificación interior del PORN y que será revisado periódicamente. Así mismo, señala que el PRUG contendrá las determinaciones precisas para el desarrollo de la normas de su competencia: normas de gestión administrativa, las de uso público, las de investigación, las de gestión, aprovechamiento y mejora de los recursos naturales, las de protección del paisaje y el patrimonio natural y un programa de actuaciones. Igualmente contendrá el desarrollo de los planes y programas a ejecutar en el Parque Regional, como son: el Plan de Investigación, los de Conservación y Restauración de los valores naturales y culturales, el de Uso Público, el de Comunicación Ambiental y el de Evaluación y Seguimiento de la gestión.

El PRUG fue redactado en 2001 pero no se llegó a iniciar los trámites para su aprobación por parte del Consejo de Gobierno de la Región de Murcia ni a presentar a la Junta Rectora del Parque.

\subsubsection{Coincidencias en la delimitación según la figura de protección}

En el Parque Regional y su entorno confluyen diversas figuras de protección con unos límites que no coinciden en tres sectores (tabla 3 y figura 2 y 3 ).

- Al Norte del canal perimetral que delimita las Salinas del resto del territorio, el sitio Ramsar Mar Menor incluye la protección hasta 200 metros; la figura de Parque regional y la de ZEPIM protegen 100 metros de los incluidos en el Sitio Ramsar, mientras que la ZEPA y el LIC terminan en el lado exterior de dicho canal perimetral.

- Charca de los baños de lodo: La declaración de Parque Regional, el Sitio Ramsar y la ZEPIM incluyen la charca de los baños de lodo, mientras que el Área de Protección de la Fauna Silvestre, la ZEPA y el LIC excluyen dicha charca.

- Puerto de San Pedro del Pinatar: La figura de Parque Regional, el Sitio Ramsar y la ZEPIM incluyen al Puerto de San Pedro del Pinatar, mientras que la declaración de Área de Protección de Fauna Silvestre, la ZEPA y el LIC, excluyen al Puerto. 
Tabla 3. Superficie y delimitación de cada figura de protección

\begin{tabular}{|c|c|c|c|}
\hline АMBITO & FIGURA & EXTENSIÓN & DELIMITACIÓN \\
\hline \multirow[t]{2}{*}{ Autonómico } & $\begin{array}{l}\text { Parque Regional } \\
\text { (incluye todo el } \\
\text { ámbito PORN) }\end{array}$ & 872,66 & $\begin{array}{l}\text { Al Norte, queda delimitado por una línea recta que, partiendo de la orilla } \\
\text { del Mediterráneo, sigue el borde externo del canal perimetral de las salinas, } \\
\text { prolongándose hacia el oeste hasta enlazar con el límite exterior de la ban- } \\
\text { da de } 100 \text { metros, medidos a partir del borde externo de dicho canal. }\end{array}$ \\
\hline & $\begin{array}{l}\text { Área de Protección } \\
\text { de la Fauna Silvestre }\end{array}$ & 841,75 & Coincide con la ZEPA y LIC \\
\hline \multirow[t]{2}{*}{ Europeo } & $\begin{array}{c}\text { Zona de Especial } \\
\text { Protección para las } \\
\text { Aves }\end{array}$ & 841,75 & $\begin{array}{l}\text { Coincide con los límites del Parque Regional, excluyendo el puerto pes- } \\
\text { quero de San Pedro del Pinatar, la zona catalogada como "resto de ámbito } \\
\text { PORN" y la laguna junto a Lo Pagán, conocida como "Charca de los Baños } \\
\text { de Lodo". También coincide con los límites del LIC }\end{array}$ \\
\hline & $\begin{array}{l}\text { Lugar de Interés } \\
\text { Comunitario }\end{array}$ & 841,75 & Coincide con la delimitación de la ZEPA. \\
\hline \multirow[t]{2}{*}{ Internacional } & $\begin{array}{l}\text { Sitio RAMSAR } \\
\text { "Mar Menor" }\end{array}$ & 882,3 & $\begin{array}{l}\text { Coincide con los límites de los humedales protegidos por la Ley 4/1992, } \\
\text { incluye la franja de } 200 \text { metros de anchura a partir del canal de drenaje } \\
\text { de las salinas, desde su intersección al norte del suelo urbano del Mojón, } \\
\text { hasta su unión, al sur, con la prolongación del límite oeste de la parcela de } \\
\text { "El Saladar", siguiente por el límite sur de ésta hasta contactar de nuevo con } \\
\text { el canal de las salinas. }\end{array}$ \\
\hline & ZEPIM & 872,66 & $\begin{array}{l}\text { Su delimitación incluye al Parque Regional de las Salinas y Arenales de San } \\
\text { Pedro del Pinatar }\end{array}$ \\
\hline
\end{tabular}

Elaboración propia.

Figura 2. Coincidencia entre las diferentes figuras de protección en el entorno de las Salinas y Arenales de San Pedro del Pinatar.

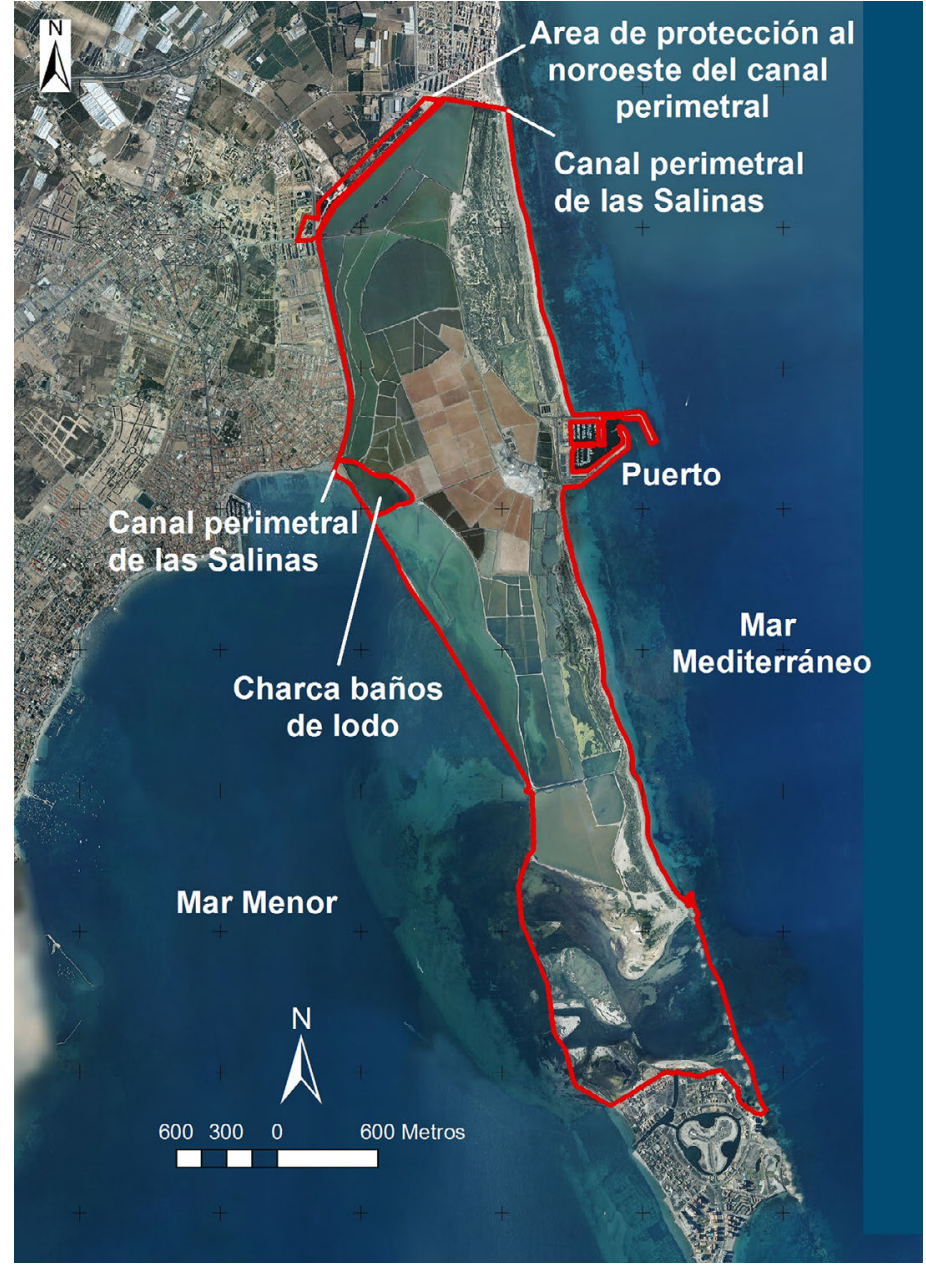

Elaboración propia. 


\subsubsection{Desarrollo de infraestructuras al noroeste del canal perimetral}

El área protegida al noroeste del canal perimetral que sirve de límite de la explotación salinera, viene determinada por los 200 metros desde dicho canal señalados por el Sitio Ramsar "Mar Menor", que incluyen los 100 metros que el PORN cataloga como "resto de ámbito PORN".

La superficie total abarca casi 29 hectáreas, donde se ha desarrollado una serie de infraestructuras que suponen una reducción de unas 7 hectáreas, sin tener en consideración la normativa del PORN y los compromisos adquiridos con el Convenio de Ramsar.

En los 100 metros denominados en el PORN como "Resto del ámbito PORN", también incluidos en el Sitio Ramsar "Mar Menor", que tiene una superficie de 15,93 hectáreas, la reducción se estima en 1 hectárea, con el desarrollo de las siguientes infraestructuras (figura 3):

1. Adecuación de un campo de futbol junto a la urbanización de El Mojón.

2. Ampliación de de la depuradora de San Pedro del Pinatar sin realizar el correspondiente Estudio de Impacto Ambiental (BORM No 16 de 21/01/2004).

3. Construcción de parte del jardín municipal "Concejal José Antonio Pérez Henarejos".

Por otro lado, dentro de los otros 100 metros incluidos dentro del Sitio Ramsar "Mar Menor" que dispone de una superficie de unas 13 hectáreas, su reducción por el desarrollo de infraestructuras se estima en unas 6,0 hectáreas, con la construcción de:

4. Desalinizadora de San Pedro del Pinatar (una parte de la misma).

5. Una pequeña superficie de la ampliación de la depuradora de San Pedro del Pinatar.

6. La avenida de las Garzas, que dé acceso a Lo Pagán y a las Salinas. En este caso se incluye también el tramo de los 100 metros dentro del "Parque Lineal", ya que el PORN contemplaba como "compatible" la construcción de la avenida.

7. Un hotel y centro de talasoterapia (una parte de los mismos)

8. Casi todo el jardín municipal "Concejal José Antonio Antonio Pérez Henarejos".

9. Algunos dúplex situados junto al Centro de Visitantes "Las Salinas".

Figura 3. Infraestructuras al noroeste del canal perimetral.

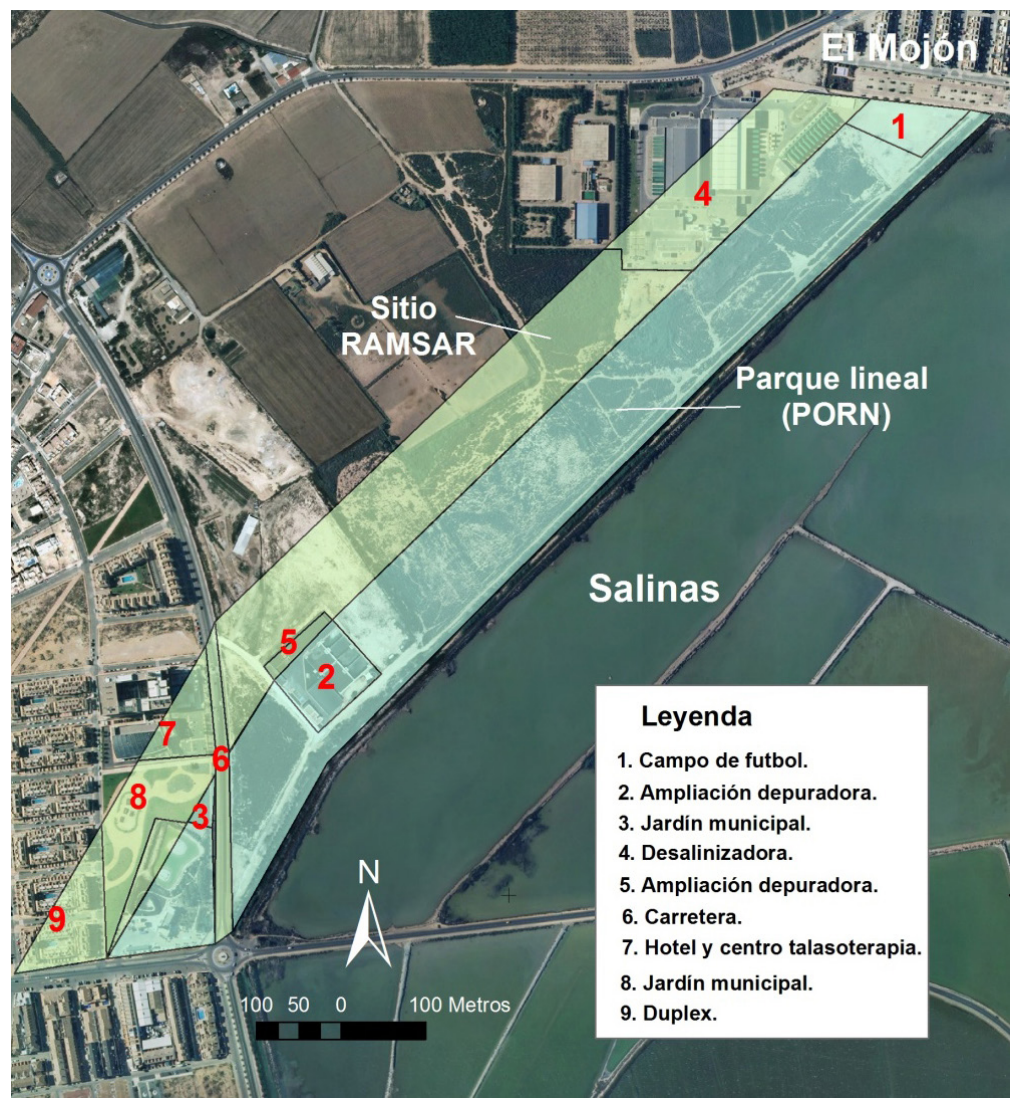

Elaboración propia. 


\subsubsection{Mantenimiento y vigilancia}

El Programa de mantenimiento y limpieza de la Red de Áreas de uso Público de la Región de Murcia se inició en la década de los 90, pero es a partir de 2003 donde se asignas recursos económicos y humanos que permiten disponer de personal en la mayor parte de los espacios naturales protegidos.

Las labores de limpieza y mantenimiento se enmarcan en un programa de apoyo a la gestión de las Infraestructuras y Âreas de Uso Público existentes en el medio natural de la Región de Murcia y en especial en la Red Natura 2000; así como a las infraestructuras básicas destinadas a la defensa forestal.

En el Parque Regional de las Salinas y Arenales de San Pedro comprenden las siguientes actuaciones de limpieza de exteriores: áreas recreativas, apartaderos, aparcamientos, senderos, itinerarios y lugares de interés más visitados.

Para el Mantenimiento y reparación básica de infraestructuras ligeras situadas en Áreas de Uso Público se dispone de una brigada de mantenimiento que resuelve todas las incidencias y solicitudes que les deriva el coordinador del servicio, relacionadas con las áreas recreativas, observatorios, aparcamientos, senderos, etc., así como otros trabajos relacionados con infraestructuras de uso público cuando se desarrollan actividades de apoyo al personal de la administración en las diferentes actividades relacionadas con la gestión y el uso público de este espacio natural.

De la vigilancia del Parque Regional se encarga un Agente de Medio Ambiente, funcionario de la Comunidad Autónoma de la Región de Murcia.

Se invierte anualmente unos 50 mil euros anuales en limpieza y mantenimiento, que ha permitido retirar entre 2003-2010 un total de 171.421 kilos de residuos sólidos urbanos, que representan una media de 21.428 kilos anuales (figura 4).

Figura 4. Residuos sólidos urbanos recogidos en el Parque Regional de las Salinas y Arenales de San Pedro del Pinatar (2003-2010)

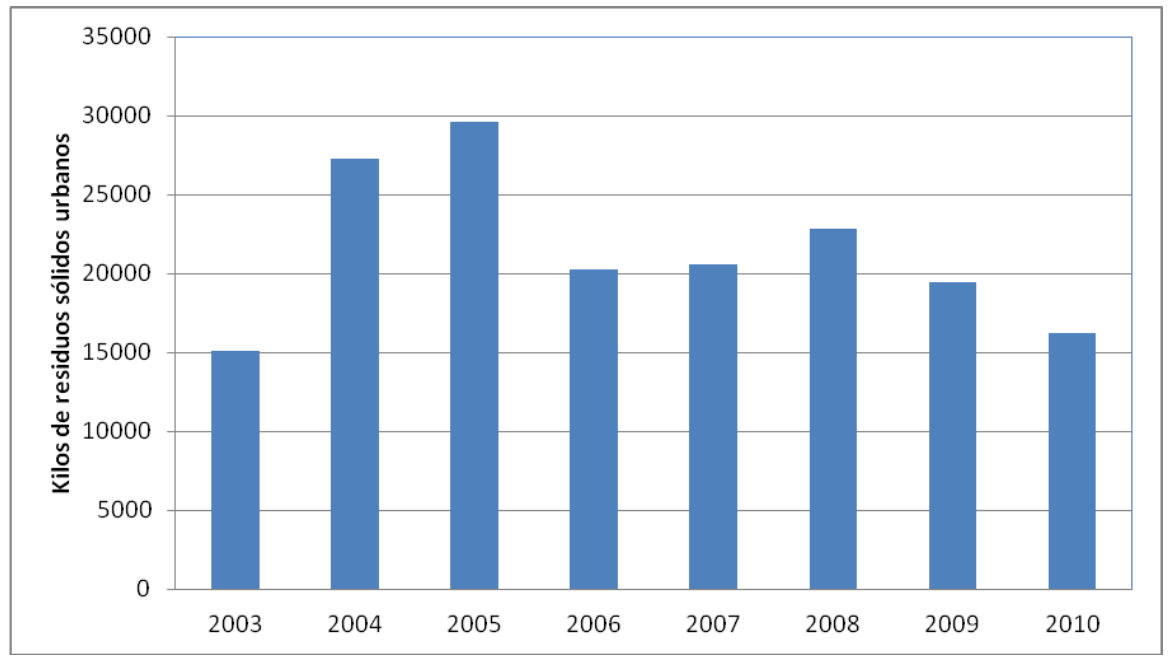

Fuente: elaborado a partir de datos suministrados por la Oficina Regional de Espacios Protegidos.

\subsubsection{Control administrativo}

Una parte importante de los esfuerzos dedicados a la conservación de este espacio natural se dedica a la elaboración de informes y autorizaciones, infracciones y denuncias y reclamaciones y sugerencias.

\subsubsection{Informes y autorizaciones}

Se incluyen los informes y autorizaciones emitidos durante el periodo estudiado tal y como recomienda De Lucio et al., (2010), incluye por un lado, las autorizaciones que son los expedientes administrativos relativos a una materia, cuya responsabilidad es propia del órgano competente en conservación de la naturaleza y, por otro, los informes que son los documentos que elabora el órgano competente en conservación de la naturaleza de forma preceptiva, cuando por razón de la materia la autorización competa a otro órgano o administración. 
La relación de informes y autorizaciones de diferente naturaleza relacionados con el Parque como son la autorización de actuaciones a diferentes administraciones y a ciudadanos y empresas. Las solicitudes de uso público han ido aumentando con el paso de los años, como son las actividades de deportes en la naturaleza, fotografía, senderismo, voluntariado, educación ambiental, infraestructuras, circulación de vehículos, etc. (tabla 4):

Tabla 4. Tramitación de informes y autorizaciones en el Parque Regional de Las Salinas y Arenales de San Pedro del Pinatar (2002-2010).

\begin{tabular}{|l|c|c|c|c|c|c|c|c|c|}
\hline & 2002 & 2003 & 2004 & 2005 & 2006 & 2007 & 2008 & 2009 & 2010 \\
\hline Informes y autorizaciones & 6 & 6 & 12 & 11 & 12 & 15 & 12 & 11 & 24 \\
\hline Solicitudes Uso Público & 7 & 6 & 7 & 7 & 10 & 14 & 25 & 25 & 33 \\
\hline
\end{tabular}

Fuente: López (2007), Ecopatrimonio (2009) y Biocyma (2010). Elaboración propia.

\subsubsection{Infracciones y denuncias}

Los agentes medioambientales denuncian anualmente a unas 2-3 personas por el tránsito y estacionamiento de vehículos de motor fuera de lugares habilitados, colocación de carteles publicitarios e inicio de obras sin autorización.

\subsection{Gestión "proactiva"}

\subsubsection{Conservación del Patrimonio Natural}

Destacan las acciones de conservación del medio biótico o abiótico, dirigidas a mantener o recuperar el estado de conservación de especies, hábitats y ecosistemas.

- Conservación de especies: Acciones "in situ" y acciones "ex situ"

Acciones "in situ": Manejo de la Gaviota patiamarilla (Larus michahellis)

La Gaviota patiamarilla (Larus michahellis) ha experimentado en el último tercio del siglo XX un rápido incremento en toda su área de distribución de la Península Ibérica, como respuesta al aumento de la disponibilidad trófica (sobre todo vertederos) y a la reducción de la presión humana en las colonias (Martí y Moral, 2003).

En Salinas de San Pedro del Pinatar el incremento de la población de gaviota patiamarilla fue continuo desde que por primera vez en 1993 nidificaran dos parejas. En 1999 con una población de 191 parejas, Ballesteros et al., (1999) constata entre otros aspectos, un descenso de la población nidificante de charrán común (Sterna hirundo) en un 64,8 \% con respecto al censo de 1995 (de 225 parejas en 1995 a 79 parejas en 1999) y en un 62,3 \% de avoceta común (Recurvirostra avosetta) con respecto a al censo de 1995 (de 151 parejas en 1995 a 57 parejas en 1999).

A esto hay que añadir los accidentes producidos en 1999 y 2000 por el vuelo rasante que las gaviotas patiamarillas sobre los trabajadores de salinera que circulan en motocicleta en sus labores de mantenimiento de la explotación salina (Ballesteros, 2006).

En el año 2000 se empieza a controlar de forma ininterrumpida la gaviota patiamarilla, impediendo su reproducción mediante la eliminación de los nidos con sus puestas en gran parte del Parque, aunque se permite su reproducción en sectores localizados y lejos de las colonias de otras especies de interés.

Las actuaciones sobre los nidos de gaviota patiamarilla ha dado como resultado:

1. Ausencia total de accidentes de los trabajadores de la salinera.

2. Recuperación del número de parejas de las especies afectadas.

3. Inicio de reproducción de otras especies escasas en la Península Ibérica, como es el charrán patinegro (Sterna sandvicensis), gaviota picofina (Larus genei) y gaviota de audouin (Larus audouinii).

Sin el control efectivo realizado de la gaviota patiamarilla, se estima que contaría con más de 1000 parejas nidificantes, por lo que es muy probable que algunas especies de elevado interés de conservación se hubieran extinguido, otras tendrían poblaciones residuales y no se hubieran instalado nuevas especies con colonias escasas en la península Ibérica. 


\section{- Acciones de conservación Ex-situ}

Las especies heridas localizadas en el Parque Regional son trasladadas al Centro de Recuperación de Fauna (CRFS) de "El Valle" (tabla 5).

Tabla 5. Especies de fauna trasladadas al centro de recuperación de fauna silvestre (1999-2010).

\begin{tabular}{|c|c|c|c|c|c|c|c|c|c|c|c|c|}
\hline & 1999 & 2000 & 2001 & 2002 & 2003 & 2004 & 2005 & 2006 & 2007 & 2008 & 2009 & 2010 \\
\hline TOTAL ESPECIES & 18 & 4 & $\begin{array}{c}\text { Sin } \\
\text { datos }\end{array}$ & 10 & 15 & 16 & 11 & $\begin{array}{c}\text { Sin } \\
\text { datos }\end{array}$ & 13 & 8 & 11 & 11 \\
\hline
\end{tabular}

Fuente: Rey-Pastor et al., (2002) y López et al., (2007, 2008, 2009 y 2010). Elaboración propia.

\section{- Conservación del paisaje y del hábitat}

Entre las actividades desarrolladas con mayor efectividad destacan los proyectos de restauración de ecosistemas litorales y dunares ejecutados en el saladar y pinar de Coterillo, playas de La Llana y Centro de Visitantes "Las Salinas", con la eliminación de especies exóticas como la uña de gato (Carpobrotus edulis), pitera (Agave amaericana) y gandul (Nicotiana glauca), la eliminación de senderos no autorizados y la repoblación con especies autóctonas como el albardín (Lygeum spartum), barrón (Ammophyllla arenaria), lentisco (Pistacea lentiscus), enebro (Juniperus macrocarpa) y sabina negra (Juniperus turbinata).

\subsubsection{Gestión del uso público}

\subsubsection{Equipamientos de Uso Público}

El Programa de Información, Atención al Visitante y Comunicación Social atiende a los visitantes en un Centro de Visitantes o Punto de Información, cuya ubicación ha ido variando con los años y en función de las necesidades. En concreto, desde su puesta en funcionamiento, se han contado con los siguientes equipamientos de atención al visitante.

Centro de Visitantes: Centro de Recursos Marinos y Humedales del Litoral, abierto todo el año entre 1997 y 2001. Centro de Visitantes "Las Salinas") Abierto todo el año desde 2002.

Puntos de Información: Punto de Información de la Playa de la Llana, abierto en verano entre 1990 y 2001. Punto de Información "Molino de la Calcetera", abierto en verano entre 2000 y 2004. Punto de Información "Charca El Coterillo", abierto en verano desde 2002.

\subsubsection{Resultados de la atención al visitante e itinerarios guiados}

En la figura 5 se contabilizan el número anual de usuarios atendidos en el Centro de Visitantes "Las Salinas" y en los puntos de información.

Figura 5. Número anual de visitantes informados. Fuente: Ballesteros et al., (2006); Rey-Pastor et al., (2002) y López et al., (2007, 2008, 2009 y 2010).

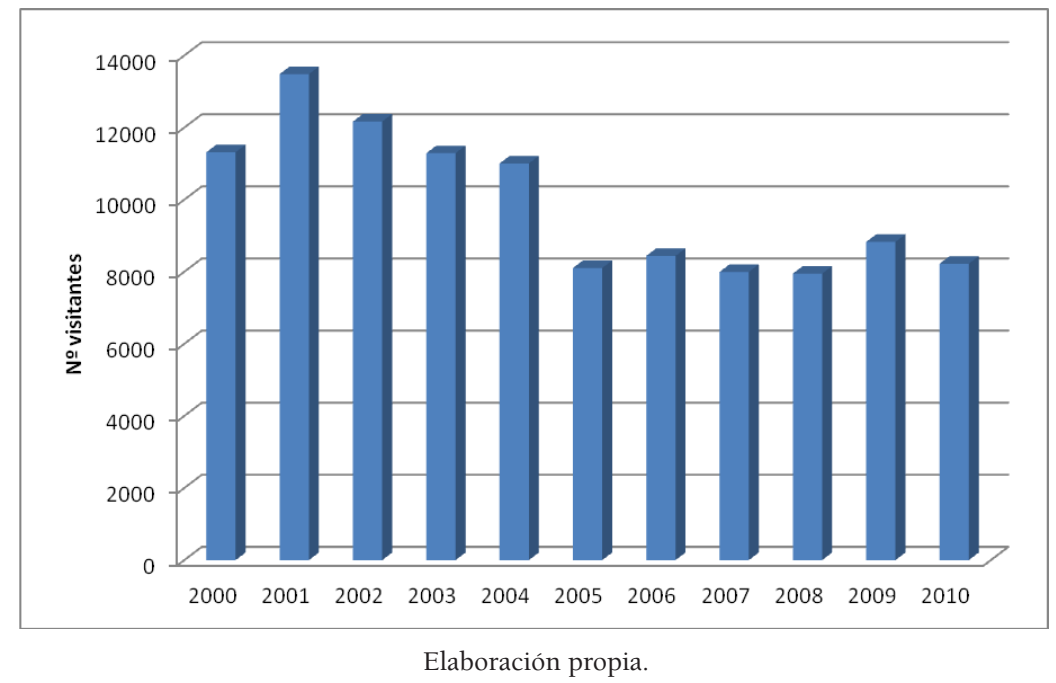


En la gráfica anterior se observa un descenso de unas 6000-7000 personas anuales en el número de visitantes atendidos a partir de 2005, ya que hasta entonces los contratos del Servicio de Información y Atención al Visitante en los espacios naturales estaban repartidos por diferentes empresas, que disponían de cierta capacidad para acordar con la administración los horarios y días de apertura al público. A partir de 2005 el contrato se asigna a una sola empresa y se acuerda con la Administración la homogeneización de todos los horarios sin tener en consideración las necesidades específicas de atención al visitante de cada espacio protegido.

Situación similar ocurre con los itinerarios guiados ofrecidos por el Servicio de Información del Parque supera el centenar de grupos, con más de 6000 personas atendidas anualmente hasta 2005. A Partir del año siguiente, el número de itinerarios no suele superar el centenar de grupos con menos de 4.000 participantes anuales.

\subsubsection{Resultados de los sondeos de opinión}

Se vienen realizando sondeos de opinión desde el inicio de los servicios de información en 1990, pero el sistema mejora la calidad con la implantación del Sistema Q de Calidad Turística en el Parque Regional en 2006, con dos tipos de encuestas, una general sobre el Parque y otra sobre el Centro de Visitantes y Servicio de Información, supervisadas por auditores externos.

Desde 2007 se vienen cumplimentando anualmente 150 encuestas generales sobre el Parque. Entre 2007 y 2010 se ha entrevistado a una media de 45,5\% de hombres y 54,5\% de mujeres, que de forma mayoritaria provienen de otras comunidades autónomas y en menor medida de la Región de Murcia. Más de la mitad de los encuestados tienen estudios universitarios y casi la mitad visitan el Parque por primera vez. Casi la mitad de los visitantes vienen en su vehículo particular, en grupo organizado o con la familia y el 88 \% dice estar "satisfecho" o "muy satisfecho".

\subsubsection{Aula de naturaleza y educación ambiental}

La administración de la Región de Murcia desarrolla un programa educativo en espacios naturales denominado "Red de Aulas de la Naturaleza", que comenzó 2004 y está dirigido de forma inicial a escolares, pero de forma progresiva se ha ido ampliando su oferta al público en general, incorporando el resto de niveles educativos, adultos y otros colectivos (Alcántar et al., 2011).

En los municipios del entorno del Parque Regional las campañas de educación ambiental tienen su origen en el año 2000 a través del Programa de Información y Atención al Visitante, cesando su actividad en 2004, coincidiendo con la puesta en funcionamiento de la Red de Aulas de la Naturaleza de la Región de Murcia.

No se disponen de datos sobre actividad educativa en centros escolares en los cursos escolares 2003/2004 y 2004/2005, pero si se tiene constancia que a partir del curso escolar 2006/2007 las actividades de educación ambiental reciben un impulso significativo con personal propio de la Red de Aulas de la Naturaleza de la Región de Murcia, que en el Parque Regional pone en marcha el Taller de Naturaleza "Molino de Quintín", en donde han participado anualmente unos 1.200 alumnos de más de 40 centros de enseñanza.

\subsubsection{Actuaciones y mejoras en los equipamientos de Uso Público}

La Administración Regional destina en sus presupuestos anuales unos fondos para actuaciones que redunden en la mejora del estado de las infraestructuras y equipamientos de uso público. Dichos fondos, pueden provenir tanto de presupuestos propios, como de proyectos cofinanciados por la Unión Europea $\mathrm{u}$ otras administraciones o entidades.

Entre las actuaciones más representativas ejecutadas entre 2001 y 2010 destaca la instalación de paneles sobre la normativa del Parque, interpretación de los hábitats, la flora y la fauna, ampliaciones y mejora de las infraestructuras del Centro de Visitantes, obras en las pasarelas de acceso a las playas, elaboración de contenidos para las audio-guías del Parque, adecuación de senderos, remodelación de aparcamientos.

\subsubsection{Certificado Q de Calidad Turística}

La Q de Calidad Turística es una certificación desarrollada por EUROPARC-España que, en el caso de espacios naturales, acredita que un espacio protegido tiene unos servicios e instalaciones de Uso Público 
de calidad, compatibles con los objetivos de conservación y fue implantada en las Salinas de San Pedro del Pinatar en 2006.

Esta norma está vinculada a diez aspectos concretos de Uso Público: dirección, acogida y recreación, información, señalización, educación ambiental e interpretación del patrimonio, reservas, seguridad, limpieza y mantenimiento, gestión ambiental, y seguimiento y evaluación (Dirección General de Medio Natural 2006).

El compromiso por la Calidad va más allá de la Certificación, tras la que se debe realizar una auditoría de seguimiento anualmente y una renovación cada dos años.

\subsubsection{Reclamaciones y sugerencias}

Con las hojas de sugerencias el público visitante puede expresar sus inquietudes, quejas e incluso felicitaciones, por determinadas actuaciones o situaciones que se lleven a cabo en el Parque Regional Salinas de San Pedro.

Entre 2007-2010, del total de inquietudes recogidas por el Servicio de Información del Parque, el $50,6 \%$ son quejas, el $37,8 \%$ son sugerencias y el $11,6 \%$ son felicitaciones.

\subsection{Mejora del conocimiento}

\subsubsection{Colaboración y participación en Proyectos de investigación}

La Administración promueve programas de seguimiento y en otros, son las universidades o centros de investigación quienes desarrollan sus propios estudios.

En 2002, construido con fondos europeos, se inauguró el Centro de Investigación y Conservación de Humedales "Las Salinas", con el objetivo de fomentar la investigación sobre humedales, en él se ubicó el Programa de Seguimiento Biológico de Aves Acuáticas y humedales y se intentó desarrollar algún proyecto de investigación, si bien, la investigación sobre humedales no fue dotada con presupuesto propio, por lo que en la práctica su funcionamiento fue muy limitado, escaso y breve en el tiempo.

\subsubsection{Programa de Seguimiento Biológico}

El Programa de Seguimiento Biológico desarrolla de forma continuada un análisis de la evolución de las poblaciones de aves acuáticas, a la vez que identifica los problemas y amenazas para su conservación.

En el espacio protegido se han citado más de 160 especies de aves, de las que unas 126 especies tienen una presencia regular. Destacan las colonias reproductoras de larolimícolas, algunas de las cuales han propiciado que las Salinas de San Pedro hayan sido designadas como ZEPA, tal es el caso de la avoceta común (Recurvirostra avosetta) que mantiene entre 50-100 parejas, la cigüeñuela común (Himantopus himantopus) con 30-40 parejas, el Charrancito común (Sterna albifrons) con unas 200-300 parejas y la Pagaza piconegra (Gelochelidon nilotica) con unas 150-200 parejas.

Con respecto al resto de vertebrados, destaca la presencia del fartet (Aphanius iberus), pez endémico del levante y sur de la Península Ibérica que se encuentra amenazado, el eslizón ibérico (Chalcides bedriagai) y el camaleón común (Chamaleo chamaleon) que tiene una densidad de 158,75 camaleones/ha, una de las más altas de España.

\subsection{Participación social}

En este apartado se incluyen las actuaciones desarrolladas por la Administración:

\subsubsection{Junta Rectora del Parque}

Según el artículo 92 del PORN, la Junta Rectora es el órgano de participación y de colaboración en la gestión del Parque Regional Salinas y Arenales de San Pedro del Pinatar, en los términos previstos en el Decreto 9/1994, de 4 de febrero (BORM nº 36, de 14.02.1994), de constitución y funcionamiento de Juntas Rectoras de Espacios Naturales Protegidos, modificado por Decreto 2/1995, de 3 de febrero (BORM n ${ }^{\circ}$ 43, de 21.02.95).

Desde la aprobación del PORN en 1995, la Junta Rectora se ha reunido en cuatro ocasiones, la primera en mayo 1996, otra en octubre de 1997, la tercera en diciembre de 2000 y la última en junio de 2001. 


\subsubsection{Programa de Voluntariado Ambiental}

El comienzo del voluntariado ambiental en este Parque Regional se remonta a 1996 en el marco del Programa de Información y Atención al Visitante.

En 2003 nace el Proyecto de Acción de Voluntariado Ambiental "Artemia", que desarrolla sus actuaciones en este Parque Regional, que incluye, entre otras acciones, tareas de ayuda a la nidificación de aves, mejora de hábitats mediante repoblaciones de especies autóctonas y colaboración en la erradicación de las alóctonas, anillamiento científico de aves, ayuda al Programa de Información y al mantenimiento puntual de éste espacio.

Con las acciones desarrolladas durante estos años se han conseguido alcanzar, entre otros, los siguientes objetivos (López et al., 2007; 2008, 2009 y Navarro et al., 2011):

- Favorecer la supervivencia y conservación de especies de fauna y flora, con acciones destinadas a la mejora de hábitat y eliminación de especies vegetales exóticas.

- Estudiar la flora y fauna con la realización de censos y avistamientos.

- Colaborar en acciones de sensibilización ambiental con el Programa de Información del Parque, y en la celebración de días especiales.

- Complementar las tareas de mantenimiento con las de reparación de vallas, acondicionamiento del Jardín del Centro de Visitantes y otras.

Según Navarro et al., (2011) las acciones realizadas tienen un impacto positivo y directo para las especies (censos, anillamiento, elaboración de cajas nido y huras) y vegetación (retirada de especies alóctonas, reforestación con autóctonas), así como para las infraestructuras (eliminación de basuras, arreglo de vallados) y para los propios visitantes (elaboración de de paneles de flora y participación en la celebración de Días Mundiales).

El Proyecto de Voluntariado "Artemia" ha organizado entre 2003-2010 una media de 13,5 actividades anuales, con una participación media de 6 voluntarios/actividad.

\subsection{Dinamización social}

El Equipo de Información fomenta y participa en aquellas actividades relevantes del Parque Regional y su entorno. Además, promueve la implicación de la población, y en especial la población local, en el conocimiento y conservación del Espacio Protegido.

\subsubsection{Participación en medios de comunicación}

La difusión en los medios de comunicación, sobre todo en el ámbito local, ha tenido como objetivo principal la de difundir y sensibilizar a la población del entorno sobre los valores naturales del Parque, fomentar la participación social en las iniciativas emprendidas por el voluntariado ambiental y publicitar los eventos más relevantes.

\subsubsection{Colaboración con otras administraciones, organizaciones y empresas}

Otras de las tareas de dinamización social ha sido mantener una comunicación fluida con las diferentes concejalías del ayuntamiento de San Pedro del Pinatar, con el fin de desarrollar acciones conjuntas en diferentes ámbitos relacionados con la conservación.

Así mismo, se ha fomentado la colaboración con otras administraciones, empresas y organizaciones no gubernamentales para el desarrollo de diferentes actividades, como es publicar artículos y envío de noticias en revistas, entrega de material divulgativo, cumplimentación de informes sobre diferentes aspectos de Parque y el uso público.

\subsubsection{Acciones formativas del personal del Parque}

Desde 2007 se diseña anualmente para el personal del Programa de Información y de Aulas de la Naturaleza una serie de actividades de formación continua entre las que destacan: interpretación del patrimonio, educación ambiental, dinamización social, etc. (López, 2010). Enttre 2007 y 2010 se han organizado entre 6 y 12 cursos de formación.

\section{CONCLUSIONES}

La evaluación de la gestión es uno de los grandes retos a los que se enfrentan los espacios naturales protegidos. La planificación y gestión de las Salinas de San Pedro debe estar basada en el mantenimiento 
sostenible de la relación entre la naturaleza y las diversas actividades económicas que se desarrollan en su interior.

Dentro de la evaluación de la gestión, se evalúa, por un lado la "eficacia", es decir, si la gestión ha alcanzado determinados objetivos y por otro, se realiza una aproximación a la "eficiencia", es decir, relación entre coste-objetivos.

Con respecto al funcionamiento básico, el desarrollo de los instrumentos de ordenación y planificación 15 años después de la aprobación del PORN, presenta un desarrollo inferior a la mitad del total de los artículos que plantean actuaciones concretas. Se han ejecutado de forma exitosa proyectos de control de especies vegetales exóticas y revegetación con especies autóctonas, se dispone de un Servicio de Información al Visitante con profesionales muy cualificados, de un Centro de Interpretación bien dotado y se ha conseguido recuperar la actividad pesquera tradicional de las Encañizadas. Por otro lado, entre los artículos que no se han puesto en marcha destaca la aplicación de los planes de recuperación de la fauna y flora. La recuperación y conservación de elementos de Interés Cultural. Tampoco se ha asignado un presupuesto anual para el desarrollo del PRUG, una vez que sea aprobado. No se fomenta la participación pública mediante reuniones anuales de la Junta Rectora y no se dispone de un Convenio con la Compañía Salinera. En éste espacio protegido confluyen seis figuras de protección que tienen un ámbito autonómico, europeo e internacional, con unos límites que no coinciden al norte del canal perimetral, en la charca de los Baños de Lodo y en el Puerto de San Pedro.

De la denominada Gestión "proactiva" está teniendo un excelente resultado el control anual de gaviota patiamarilla, que garantiza la ausencia de riesgo de accidentes laborales de trabajadores de salinera, el mantenimiento y la ocupación de motas salineras por parte de un número cada vez mayor de especies de larolimícolas coloniales. Para la conservación del paisaje y del hábitat se han ejecutado proyectos exitosos en el camino de los Molinos y en la Charca de los Baños de Lodo. Sin embargo, se ha dejado al margen la ordenación y gestión territorial a la periferia del Parque, con un desarrollo urbanístico en los límites terrestres externos al área protegida entre el Mojón y Lo Pagán, interrumpida en el sector central, con el desarrollo de infraestructuras de carácter industrial (desaladora y desalobrizadora), donde aún subsisten algunas parcelas agrícolas y saladares marginales. Se ha perdido una hectárea incluida en el PORN por la ampliación de la depuradora de San Pedro del Pinatar, sin el correspondiente Estudio de Impacto Ambiental y sin ser consultada la Junta Rectora del Parque; la adecuación de un campo de futbol junto a la urbanización del Mojón y casi todo el jardín municipal "Concejal José Antonio Pérez Henarejos.

De la Gestión del uso público y educación ambiental, el Servicio de Información se ha convertido en uno de los ejes principales de la gestión del Parque por su cercanía a la población local y a los turistas, ya que atiende a más de 8.000 visitantes al año, organiza cerca de un centenar de itinerarios ambientales en los que participan unas 3.000 personas, organiza actividades especiales los días mundiales y desarrolla sondeos de opinión.

Con respecto a la mejora del conocimiento, la Administración Regional promueve un programa de seguimiento de aves acuáticas, con estimas de tendencias de especies.

La Participación social es escasa, en 15 años que tiene de vigencia el PORN, la Junta Rectora se ha reunido solamente en cuatro ocasiones, la última vez en 2001.

La dinamización social presenta unos buenos resultados, sobre todo en mantener una estrecha colaboración con los actores principales entre los que destacan el Ayuntamiento de San Pedro del Pinatar, Asociación de Vecinos del Mojón y Salinera Española.

\section{BIBLIOGRAFÍA}

BAllesteros, G.A., BARBERÁ, G., PAVÍA, A. y PRIETO, A. (1999): Elaboración de Directrices de Protección de Fauna en el Parque Regional de las Salinas y Arenales de San Pedro del Pinatar y Paisaje Protegido de los Espacios Abiertos e Islas del Mar Menor. Cota Ambiental-Consejería de Agricultura, Agua y Medio Ambiente. Murcia, 231 pp.

BALlesteroS, G.A. (2006): Análisis del estado de conservación del Parque Regional de las Salinas y Arenales de San Pedro del Pinatar. Murcia: Cota Ambiental S.L.-Consejería de Industria y Medio Ambiente. Murcia, 14 pp.

MARTÍ, R. y MORAL, J.C. (2003): Atlas de de las Aves Reproductoras de España. Dirección General de Conservación de la Naturaleza-Sociedad Española de Ornitología. Madrid, 452 pp. 
BIOCYMA. Memoria Anual de Gestión (2010): Parque Regional de las Salinas y Arenales de San Pedro del Pinatar. BIOCYMA-Consejería de Agricultura y Agua de la Región de Murcia. Murcia, 2010. 47 pp.

CONSEJO DE EUROPA (2000): European Landscape Convention. Consejo de Europa. Estrasburgo.

CONSEJO REGIONAL DE ESPACIOS NATURALES DE CASTILLA Y LEÓN (2007): Informe anual sobre estrategia y resultados de la gestión de los Espacios Naturales Protegidos en Castilla y León 2006. Consejería de Medio Ambiente. Junta de Castilla y León. Valladolid, 87 pp.

DE LUCIO, J.V., ATAURI, J.A., MUÑOZ, M., MÚGICA, M., PUERTAS. (2010): Herramientas para la Evaluación de Áreas Protegidas: Modelo de Memoria de Gestión. Manual08. EUROPAC-España. Madrid, $123 \mathrm{pp}$.

DOUVERE, F. (2008): The importance of marine spatial planning in advancing ecosystem-based sea use management, Marine Policy, 32, pp. 762-771.

DGA (2004): La Gestión de los Espacios Naturales Protegidos de Aragón. Memoria divulgativa 2004. Dirección General del Medio Natural. Departamento de Medio Ambiente. Diputación General de Aragón. Zaragoza, 201 pp.

DIRECCIÓN GENERAL DE MEDIO AMBIENTE. (1997): Boletín Informativo ${ }^{o} 3$ del Parque Regional de las Salinas de San Pedro del Pinatar y humedales protegidos de la Región de Murcia n 3, Consejería de Medio Ambiente, Agricultura y Agua. Murcia, 8 pp.

DIRECCIÓN GENERAL DEL MEDIO NATURAL (2003): Plan Rector de Uso y Gestión del Parque Regional de las Salinas y Arenales de San Pedro del Pinatar, Consejería de Agricultura, Agua y Medio Ambiente. Murcia, 256 pp.

DIRECCIÓN GENERAL DEL MEDIO NATURAL (2006): Boletín El Mirador de la Red de Espacios Protegidos de la Región de Murcia no 17, Consejería de Industria y Medio Ambiente. Murcia, 7 pp.

ECOPATRIMONIO (2009): Memoria Anual de Gestión 2009: Parque Regional de las Salinas y Arenales de San Pedro del Pinatar. Dirección General de Patrimonio Natural y Biodiversidad, Consejería de Agricultura y Agua de la Región de Murcia. Murcia, 38 p.

GARCÍA, L. y HUERCO, E. (2007): El sistema de indicadores de desarrollo sostenible de los parques naturales del Principado de Asturias (INDES-PAR). Boletín EUROPARC-España, pp. 27-32.

GASTON, K.J., JACKSON, S.F., CANTÚ-SALAZAR, L., CRUZ PIÑÓN, G. (2008): The Ecological Performance of Protected Areas. Annual Review of Ecology, Evolution and Sistematics, n 39, pp. 93-105.

LÓPEZ, T. (2007): Memoria de gestión del Parque Regional de las Salinas y Arenales de San Pedro del Pinatar. 2006-2007. Dirección General de Medio Natural. Murcia. 79 pp.

LÓPEZ, T., DOMINGUEZ, S., MORENO, M.A., RAMOS, F. y SAURA, D. (2008): Programa de Información, Atención al Visitante y Comunicación Social. Parque Regional de las Salinas y Arenales de San Pedro del Pinatar. Memoria anual.Consejería de Agricultura y Agua. Murcia. 104 p.

LÓPEZ, T., RAMOS, F., SAURA, D., GARCÍA, J., CASTEJÓN, N., MORENO, M.A. y GÓMEZ, M.E. (2009): Programa de Información, Atención al Visitante y Comunicación Social. Parque Regional de las Salinas y Arenales de San Pedro del Pinatar. Memoria anual 2009. Consejería de Agricultura y Agua. Murcia, 122 pp.

LÓPEZ, T., NORTE, M.J., CASTEJÓN, N., GOMEZ, M.E., RAMOS, F. y SAURA, D. (2010): Programa de Información, Atención al Visitante y Comunicación Social. Parque Regional de las Salinas y Arenales de San Pedro del Pinatar. Memoria anual 2010. Murcia: Consejería de Agricultura y Agua. Murcia,122 pp.

MALLARACH, J.M., GERMAIN, J., COMAS, E., SABATÉ, X, BASORA, X. i CRUANYES, J. (2008): Protegits, de fet o de dret? Primera avaluació del sistema d'espais naturals protegits de Catalunya. Institució Catalana d'Historia Natural. Barcelona, 112 pp.

NAVARRO, A., GONZÁleZ, D. y LÓPEZ, J.D. (2011): Programa de Voluntariado Ambiental en Espacios Naturales Protegidos de la Región de Murcia. Informe final 2010-2011. Consejería de Presidencia. Murcia, $200 \mathrm{pp}$.

PULLIN, A.S., KNIGHT, T.M., STONE, D.A.and CHARMAN, K. (2004): Do conservation managers use scientific evidence to support their decision-making? Biological Conservation, 2004, $\mathrm{n}^{\mathrm{o}} 119$, pp. 245-252.

UNEP-WCMC (2008): State of the world's protected areas: an anual review of global conservation progress. UNEP-WCMC. Cambridge, 68 p. 